\title{
VEGF mRNA and protein concentrations in the developing human eye
}

Irene T. Ma', Suzanne McConaghy ${ }^{2}$, Kopperuncholan Namachivayam³ ${ }^{3}$ Brian A. Halloran ${ }^{4}$, Ashish R. Kurundkar4, Krishnan MohanKumar ${ }^{3}$, Akhil Maheshwari ${ }^{3,5}$ and Robin K. Ohls ${ }^{2}$

BACKGROUND: Vascular endothelial growth factor (VEGF), a well-characterized regulator of angiogenesis, has been mechanistically implicated in retinal neovascularization and in the pathogenesis of retinopathy of prematurity. However, the ontogeny of VEGF expression in the human fetal retina is not well known. Because retinal vasculature grows with gestational maturation, we hypothesized that VEGF expression also increases in the midgestation human fetal eye as a function of gestational age.

METHODS: To identify changes in VEGF gene expression during normal human development, we measured VEGF mRNA by quantitative PCR and measured VEGF protein by enzymelinked immunosorbent assay and western blots in 10-24 wk gestation fetal vitreous, retina, and serum.

RESULTS: VEGF mRNA expression in the retina increased with gestational age. VEGF isoform A, particularly its VEGF ${ }_{121}$ splice variant, contributed to this positive correlation. Consistent with these findings, we detected increasing $\mathrm{VEGF}_{121}$ protein concentrations in vitreous humor from fetuses of 10-24 wk gestation, while VEGF concentrations decreased in fetal serum.

CONCLUSION: VEGF $_{121}$ mRNA and protein concentrations increase with increasing gestational age in the developing human retina. We speculate that VEGF plays an important role in normal retinal vascular development, and that preterm delivery affects production of this vascular growth factor.

nfants born at the limits of viability are susceptible to morbidities involving many organ systems. Retinopathy of prematurity (ROP) is a well-known morbidity specific to the developing eye. This disease involves the abnormal maturation of the retinal vasculature, and it is one of the most common causes of irreversible childhood blindness today (1). Although epidemiological evidence indicates ROP to be associated with multiple risk factors such as prematurity, supplemental or prophylactic oxygen use, low birth weight, infections, and poor postnatal weight gain, the etiopathogenesis of this disorder remains unclear (1-5).

Vascular endothelial growth factor (VEGF), a well-characterized regulator of angiogenesis, has been mechanistically implicated in retinal neovascularization and in the pathogenesis of ROP (6-9). Understanding the ontogeny of VEGF expression in the normal human fetal retina is an important step in the study of angiogenic factors operative in ROP $(10,11)$. In this study, we hypothesized that VEGF expression increases in the midgestation human fetal eye as a function of gestational age, and measured VEGF isoforms and splice variants in retinal tissue and vitreous fluid obtained from fetuses of $10-24 \mathrm{wk}$ gestation.

\section{RESULTS}

Endogenous Controls

We evaluated $\beta$-actin, glyceraldehyde 3-phosphate dehydrogenase (GAPDH), hypoxia inducible factor $1 \alpha$ (HIF-1 $\alpha$ ), and ribosomal $18 \mathrm{~s}$ as possible genes to serve as normalizing controls in quantitative PCR (qPCR) reactions (Figure 1). We found no statistical difference in gene expression across all gestational ages tested $(10-24 \mathrm{wk})$ with GAPDH $(P=0.108)$, $\beta$-actin $(P=0.522)$, or HIF- $1 \alpha(P=0.077)$, while 18 s gene expression increased significantly from early to late mid-gestation $(P=0.006)$. We chose GAPDH to serve as our endogenous control because of its relative abundance in relation to VEGF.

\section{VEGF Concentrations in Serum and Vitreous}

We initially measured VEGF concentrations in vitreous and serum in 10-24 wk fetal samples (Figure 2). The enzymelinked immunosorbent assay (ELISA) primarily measured VEGF $_{165}$ but could not distinguish VEGF Vrom $_{165}$ fEGF ${ }_{121}$. Serum $\mathrm{VEGF}_{165 / 121}$ concentrations were significantly higher at 10-14 wk gestation than concentrations at other gestational ages, and were higher than vitreous concentrations at $10-14 \mathrm{wk}$ $(P<0.05)$. Vitreous $\mathrm{VEGF}_{165 / 121}$ concentrations were similar among gestational age groups, and were similar to serum concentrations at $15-17,18-21$, and 22-24 wk gestation.

\section{VEGF-A Expression Increases in the Second Trimester Retina With Advancing Gestation}

We measured mRNA expression of VEGF-A, -B, -C, and -D isoforms in fetal retinal tissue by RT-qPCR. As shown in Figure 3, the expression of VEGF-A, but not VEGF-B, -C, or 
a

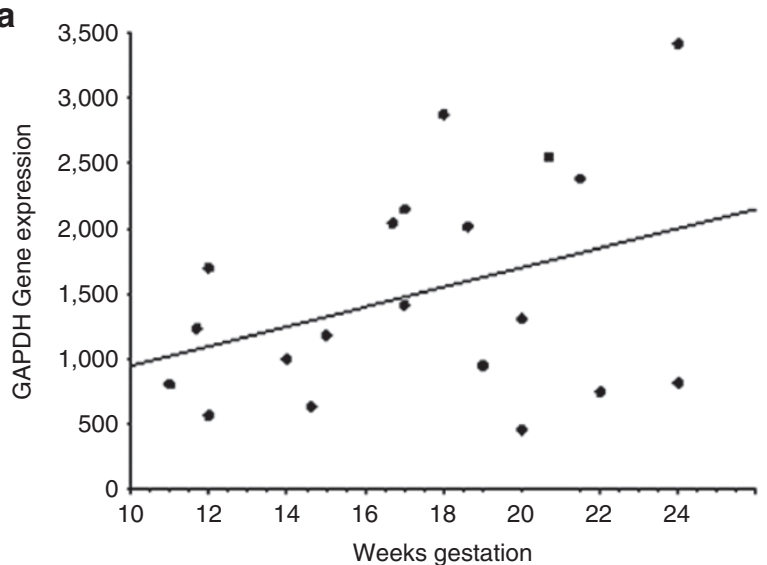

C

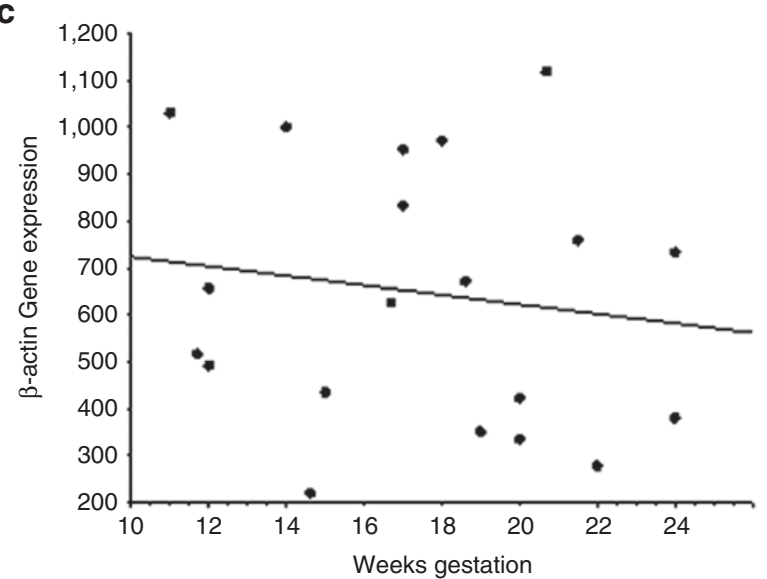

b

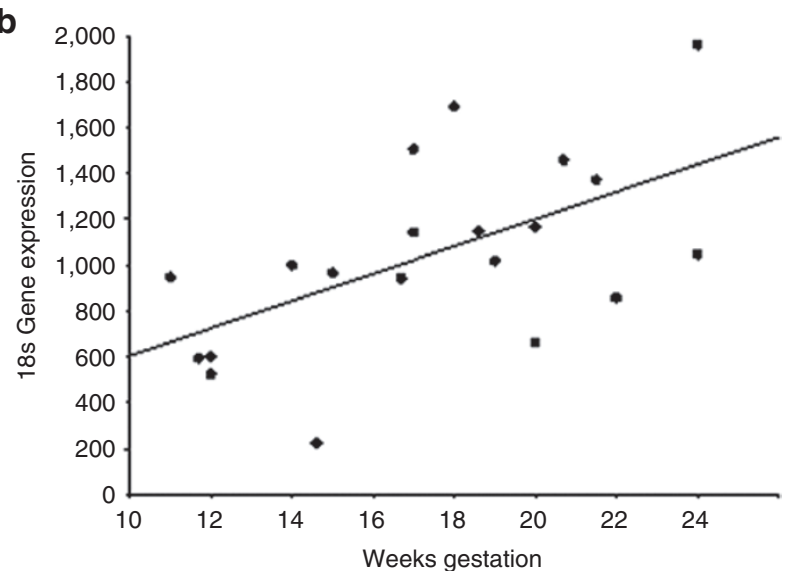

d

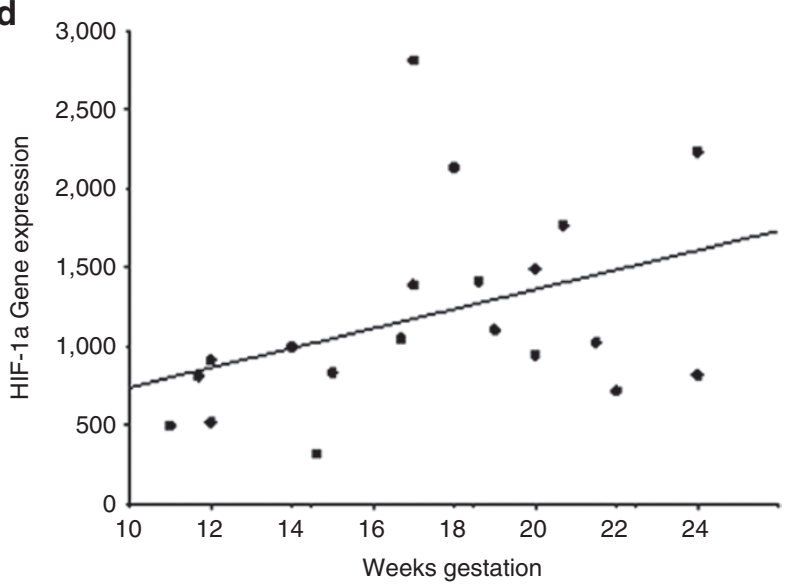

Figure 1. Gene expression of glyceraldehyde 3-phosphate dehydrogenase (GAPDH), $\beta$-actin, 18s, and HIF-1 $\alpha$. Gene expression (expressed as ng/ $\mu$ l starting total RNA) in retina for GAPDH (panel a), 18s (panel b) $\beta$-actin (panel c), and HIF-1 $\alpha$ (panel d) from 10-24 wk samples is shown. Gene expression of $\operatorname{GAPDH}\left(R^{2}=0.18\right)$, $\beta$-actin $\left(R^{2}=0.22\right)$, and hypoxia inducible factor $(\mathrm{HIF})-1 \alpha\left(R^{2}=0.16\right)$ was not significantly different across the range of gestational age tested, while gene expression of $18 \mathrm{~s}$ increased with increasing gestational age $\left(R^{2}=0.33 ; P=0.006\right)$.

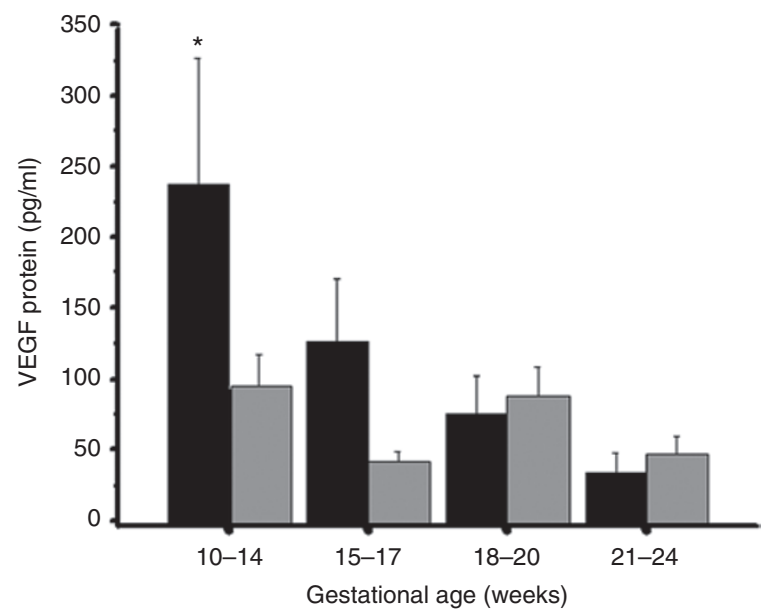

Figure 2. Vascular endothelial growth factor (VEGF) protein concentrations in fetal serum and vitreous. VEGF ${ }_{165 / 121}$ protein concentrations in serum (black bars) and vitreous (gray bars) measured from 10 to $24 \mathrm{wk}$ gestation. VEGF $_{165 / 121}$ protein concentrations were significantly greater in serum at $10-14 \mathrm{wk}\left({ }^{*} P<0.05\right)$ than in serum at other gestational ages, while concentrations in vitreous were similar at all gestational ages tested. Serum VEGF ${ }_{165 / 121}$ protein concentrations were similar to vitreous concentrations from 15 to $24 \mathrm{wk}$.
$\mathrm{D}$, increased in the midgestation retina as a function of gestation age. VEGF mRNA expression showed a strong positive correlation with gestational age (inset; Spearman's $r=0.77, P$ $<0.05)$. Consistent with these findings, we measured VEGF in retinal tissue and showed higher VEGF-A protein expression in tissue from 20 to $24 \mathrm{wk}$ fetuses than 11 to $14 \mathrm{wk}$ fetuses (Figure 4).

\section{VEGF $_{121}$ Is the Predominant Splice Variant of VEGF-A in the Midgestation Fetal Retina}

We next PCR-amplified major splice variants of VEGF-A mRNA in retinal tissue from fetuses of 20-24 wk gestation. As shown in Figure 5, VEGF ${ }_{121}$ was the predominant splice variant of VEGF-A, but we also detected faint bands of VEGF ${ }_{165}$ and $\mathrm{VEGF}_{189}$. These findings were specific to the retina; we detected only VEGF ${ }_{121}$ and $\mathrm{VEGF}_{206}$ in the fetal brain, but all the five splice variants in the fetal colon at the same gestational age.

We next used our SYBR green I-based qPCR protocol to measure the developmental changes in $\mathrm{VEGF}_{121}, \mathrm{VEGF}_{165}$, and $\mathrm{VEGF}_{189}$. Although all the three splice variants showed increased expression with advancing gestation, $\mathrm{VEGF}_{121}$ and $\mathrm{VEGF}_{165}$ showed a greater developmental change than $\mathrm{VEGF}_{189}$ (Figure 6). 


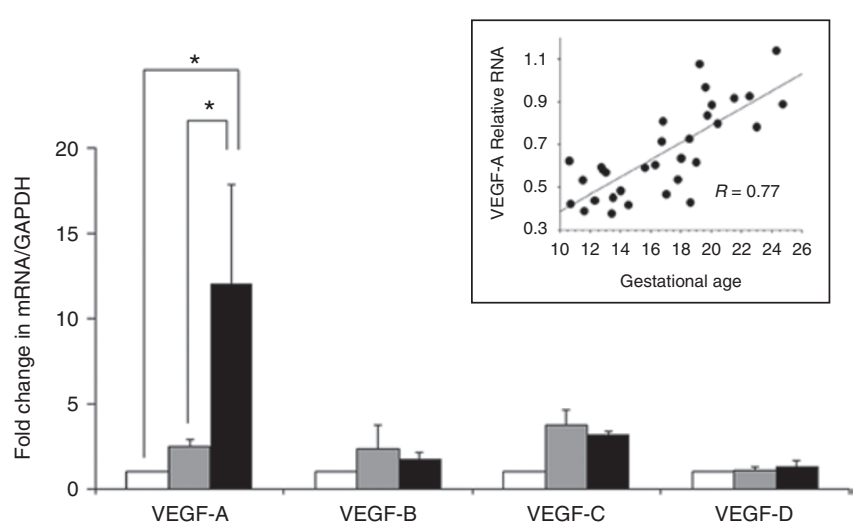

Figure 3. Vascular endothelial growth factor (VEGF)-A mRNA expression in the second trimester retina with advancing gestation. Bar diagrams (means $\pm \mathrm{SE}$ ) show fold change in the mRNA expression of VEGF isoforms, VEGF-A, $-B,-C$, and $-D$, in retinal tissue from fetuses of 10-14 wk (white bars), 15-19 wk (gray bars), and 20-24 wk (black bars) gestation. Inset: Scatter-plot of relative RNA expression of VEGF/18S ribosomal RNA vs. gestational age. $R=$ Spearman's correlation coefficient). ${ }^{*}$ indicates $P<0.05$.

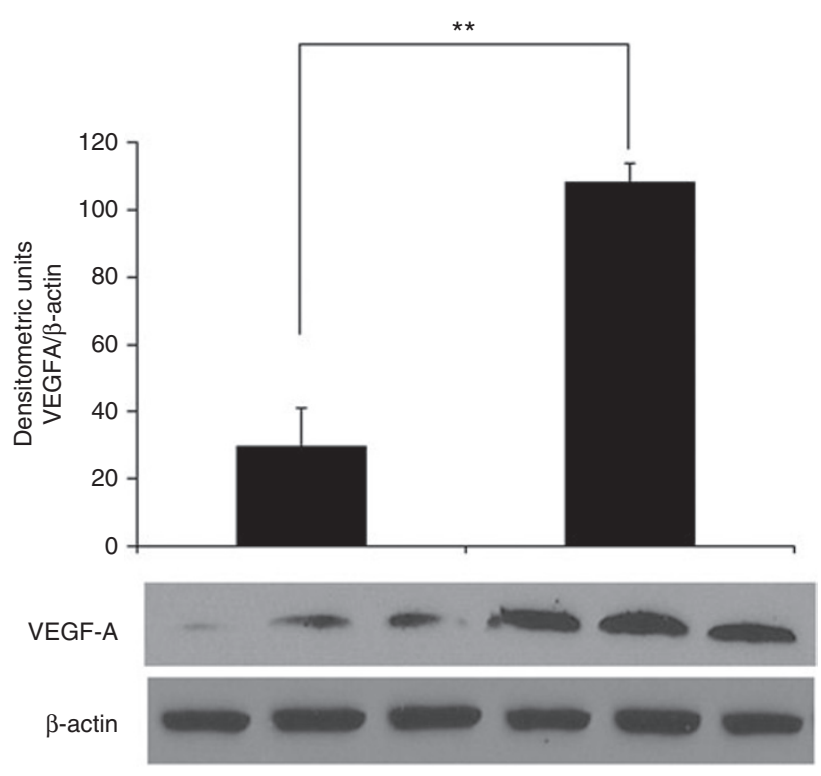

Figure 4. Tissue-bound vascular endothelial growth factor (VEGF)-A protein expression in retinal tissue from fetuses at 10-14 wk and 20-24 wk gestation. Representative Western blots show tissue-bound VEGF-A and $\beta$-actin. Bar-diagrams (means $\pm \mathrm{SE}$ ) summarize densitometric data. ${ }^{*}$ indicates $P<0.01$. 10-14 week for left column, 20-24 week for right column.

\section{VEGF Concentrations Increase in the Vitreous Humor With Advancing Gestation}

Existing information indicates that $\mathrm{VEGF}_{121}$ and $\mathrm{VEGF}_{165}$ are diffusible proteins, in contrast to the larger, tissue-bound VEGF $_{189}$ protein (12). Therefore, to confirm the biological relevance of our findings at the protein level, we next measured VEGF $_{121}$ concentrations in vitreous humor from these fetuses. As shown in Figure 7, VEGF ${ }_{121}$ concentrations increased in vitreous humor with advancing gestation. Compared to fetuses of 10-14 wk gestation, there was a trend toward higher vitreous VEGF $_{121}$ concentrations at $15-19 \mathrm{wk}$, and significantly higher VEGF $_{121}$ concentrations at $20-24 \mathrm{wk}$.
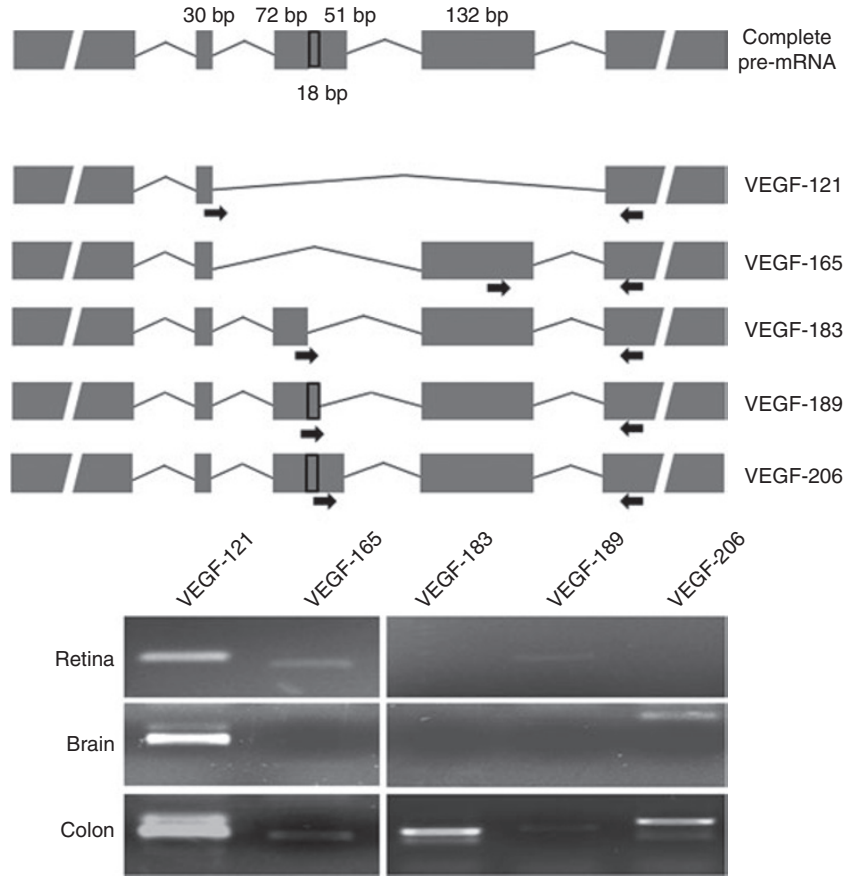

Figure 5. Vascular endothelial growth factor (VEGF)-A splice variants in the midgestation fetal retina. Schematic shows the complete pre-mRNA and the major splice variants of VEGF-A. The site of the primers is indicated by arrows (top). Representative agarose gels showing PCR products corresponding to the major splice variants in fetal retina, brain, and colon (bottom).

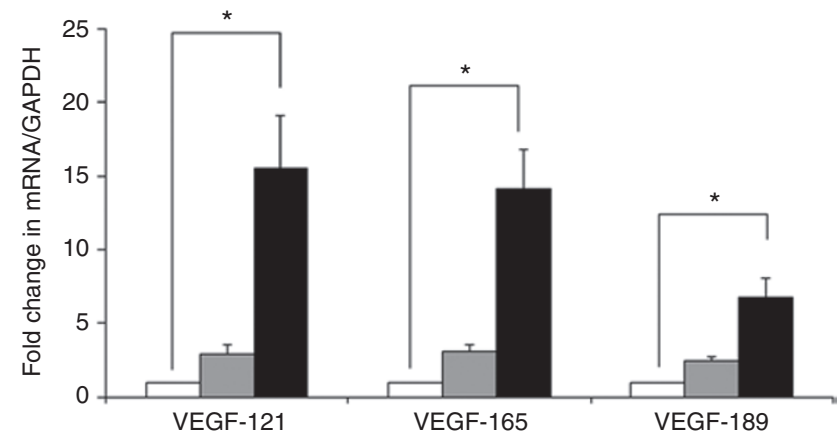

Figure 6. Developmental change in vascular endothelial growth factor (VEGF)-A splice variants in the midgestation fetal retina. Bar diagrams (means $\pm \mathrm{SE}$ ) show fold change in the mRNA expression of VEGF-A splice variants, $\mathrm{VEGF}_{121}, \mathrm{VEGF}_{165}$, and $\mathrm{VEGF}_{189}$, in retinal tissue from fetuses of 10-14 wk (white bars), 15-19 wk (gray bars), and 20-24 wk (black bars). * indicates $P<0.05$.

\section{DISCUSSION}

The normal role of VEGF in the developing human eye continues to be evaluated. In this study, we found increasing concentrations of retinal VEGF-A mRNA with increasing gestational age in the human fetal eye. $\mathrm{VEGF}_{121}$ was the predominant splice variant of VEGF-A in fetal retina, and consistent with these findings, we detected increasing VEGF $_{121}$ concentrations in vitreous humor with increasing gestational age. Our findings of increasing retinal VEGF mRNA and total VEGF protein with increasing gestational age are consistent with the developing vasculature of the eye. 


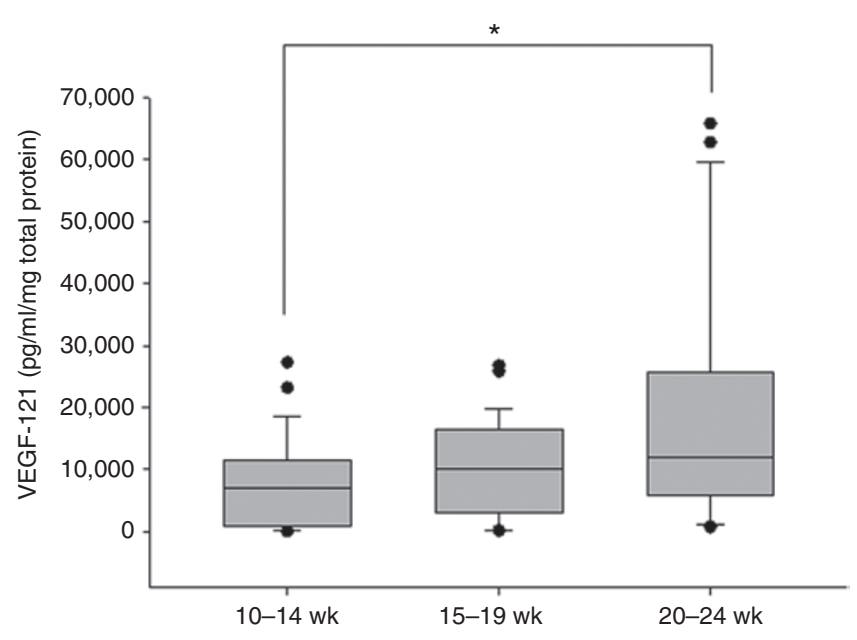

Figure 7. $\mathrm{VEGF}_{121}$ concentrations increase in the vitreous humor with advancing gestation. Box-plots show VEGF ${ }_{121}$ concentrations in vitreous humor from fetuses of 10-14, 15-19, and 20-24wk. * indicates $P<0.05$.
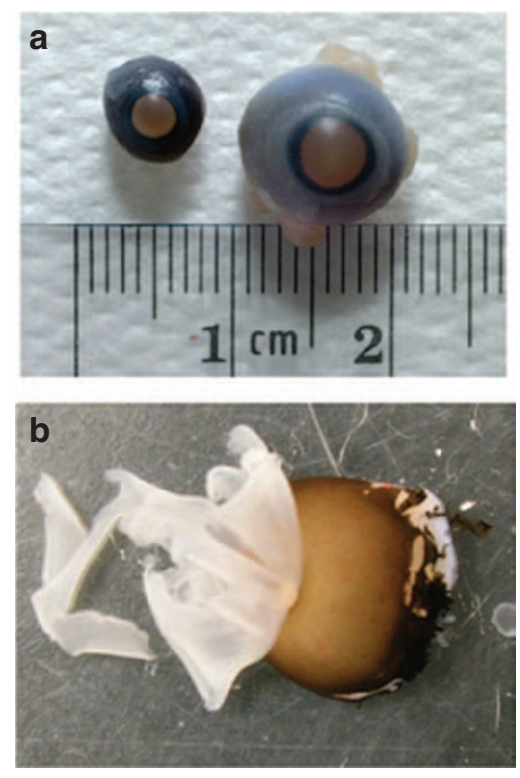

Figure 8. Fetal eyes and retina. Fetal eyes at 14 and 18 wk gestation (panel a) are shown. Dissection of the 18-wk fetal eye revealing the retina and choroid is shown (panel $\mathbf{b}$ ).

Vascularization in the eye begins during the fifth week of gestation and is not completed until after birth $(2,8)$. The eye has an initial vascular system that develops but then regresses when the permanent retinal vascular system, limited to the inner two-thirds of the retina, starts to develop in the fourth month of gestation (8). Due to its tissue origins, the retina is thought to be primarily vascularized by angiogenesis, the formation of vessels from existing vessels, rather than vasculogenesis, the de novo formation of vessels $(8,10)$. VEGF is the primary hypoxically-regulated growth factor responsible for angiogenesis, which includes formation of the hyaloid vascular system early in development as well as later retinal vessel formation. However, before it assumes its role as an angiogenic factor, VEGF may also serve as a neurogenic factor for progenitors and newly postmitotic cells in the prevascular retina (13).

The term VEGF is often used synonymously with its isoform $\mathrm{A}$. The gene is organized in eight exons $(6,14,15)$, where differential splicing results in two families of isoforms, one that is proangiogenic involved in neovascularization and the other that is antiangiogenic, inhibiting blood vessel proliferation (16). The isoforms formed from alternative splicing of the VEGF-A gene that are present in both families include at least four transcripts containing 121, 165, 189, and 206 amino acid residues. These VEGF-A isoforms have functionally unique, biological activity and are distinguished by their affinity for heparin $(9,14,17)$. VEGF-A has also been shown to be necessary for the development and stability of retinal vessels (18). The smaller isoforms, 121 and 165, are diffusible proteins; whereas, the larger isoforms, 189 and 206, have a high affinity for heparin and are bound to tissue. We detected VEGF to be the predominant splice variant of VEGF-A in the fetal retina. These findings show an interesting contrast with the adult retina, where the predominant splice variant is $\mathrm{VEGF}_{165}$ (19). Further study is needed to determine whether this developmental dichotomy in VEGF-A splice variants remains true in ROP and other retinal disorders.

The mechanisms for the observed maturational increase in VEGF expression in the developing retina are unclear. However, existing data indicate a role for several growth factors and cytokines as regulators of VEGF-A expression and splicing (20). For instance, transforming growth factor- $\beta$ (TGF- $\beta$ ) has been shown to act via the p38 MAPK to activate SR protein splicing factors such as SRp55, which binds the VEGF pre-RNA in the exon $8 \mathrm{~b}$ region (21). Similarly, insulin-like growth factor (IGF)-1 can phosphorylate ASF/SF2 (alternative splicing factor/splicing factor 2) through the SR protein kinase SRPK1 (serine/arginine domain protein kinase 1) (22). ASF/SF2 can bind to VEGF preRNA around proximal splice-site selection) and results in differential expression of various splice variants of VEGF-A. IGF-1 may also regulate VEGF expression via HIF- $1 \alpha$-dependent and -independent pathways. HIF-1 $\alpha$ is known to be abundant in hypoxic tissues with an active angiogenesis (22). While HIF-1 expression did not significantly increase with development, there was a trend. This fits with our understanding of the role of "physiological hypoxia" in retinal vessel development.

Previous studies have evaluated the concentration of VEGF protein in the eye. Gogat et al. (8) determined the distribution of VEGF on retinal sections during development and noted that VEGF transcripts had a temporal and spatial correlation with normal development of human ocular vasculature. That study did not detect VEGF mRNA in older mid-gestational tissues, but did detect VEGF protein. Kim et al. (23) evaluated the levels of VEGF mRNA transcripts and protein in samples from normal monkeys, and detected constitutive expression of VEGF, VEGFR-1, and VEGFR-2. Pierce et al. (24) showed that VEGF mRNA levels, followed by VEGF protein, increase in a mouse model with relative retinal hypoxia. Peer et al. (25) showed that abnormal retinal vessel growth in human and rabbit neovascular retinas was associated with dramatic elevations 
of retinal VEGF expression compared to control retinas. These findings are consistent with our hypothesis that VEGF has an integral role in the normal development of the human eye, and abnormal levels may be associated with ROP.

Our findings provide important physiological information on the normal expression of VEGF in the developing retina. Although the pathophysiology of ROP is not well understood because of the complexity of this disease, the role of VEGF is now widely accepted $(10,26,27)$. ROP has a well-recognized progression in the retina that is divided into two discrete, but associated stages: cessation of normal retinal angiogenesis and a subsequent hyperproliferative neovascular response to retinal ischemia where phase I occurs from 22 to $30 \mathrm{wk}$ postmenstrual age, and phase II from 31 to $44 \mathrm{wk}$ postmenstrual age $(2,28,29)$. Phase I occurs when the premature infant is exposed to relative hyperoxia, often associated with supplemental oxygen, resulting in reduced VEGF expression. Understanding these relationships between oxygen and VEGF can help improve the management of ROP, including both the prevention as well as early (i.e., in phase I) and later treatment (i.e., in phase II) (30). The role of growth factors, including erythropoietin (31,32), IGF-1 (2), and matrix metalloproteinases (33), on VEGF expression in the normal retina and in ROP needs further evaluation. The efficacy of anti-VEGF agents such as intravitreal bevacizumab in ROP has also emphasized the important pathophysiological role of VEGF in ROP (34). Identification of the specific isoforms of VEGF expressed in the developing retina could help reduce adverse effects and facilitate the development of more specific anti-VEGF agents. In the adult retina, the anti-angiogenic VEGF $_{165}$ antibody inhibits hypoxia-induced neovascularization (16), although our findings indicate a need to confirm whether $\mathrm{VEGF}_{121}$ or VEGF $_{165}$ is the predominant splice variant of VEGF-A in ROP.

\section{METHODS}

\section{Fetal Tissues}

This study was evaluated by the Human Research Review Committee at the University of New Mexico and deemed not to constitute human subject research as no identifiable human subject data were collected. Vitreous, serum, and retinal tissue samples were collected within $3-4 \mathrm{~h}$ of elective termination from fetuses between 10 and $24 \mathrm{wk}$ of gestation for measurement of VEGF mRNA and protein. Samples were divided into early, mid, and late second trimester based on distribution across the 10-24 wk gestation range. All biological specimens were handled and stored similarly accordingly to a standardized checklist. Gestational age was estimated based on fetal foot length. Gender information was not collected. The contents of the globe were extracted, retinal tissue (including choroid) isolated (Figure 8), and the aqueous and vitreous collected from both fetal eyes and combined for each sample. Vitreous samples were clarified by centrifugation at $200 \mathrm{~g} \times 5 \mathrm{~min}$ and then frozen @ -20 until the time of analysis. Retinal tissue from both eyes was triturated using serially smaller syringes in a commercially-available lysis buffer (T-PER tissue protein extraction reagent with added protease inhibitors; Thermo Scientific, Rockford, IL) for measurement of total protein concentration by the Bradford assay (Thermo Scientific).

\section{Western Blots}

Tissue-bound VEGF-A protein was measured in retinal samples in Western blots using a mouse monoclonal $\operatorname{IgG}_{1}$ that detects the -189 , -165 , and -121 amino acid splice variants (Santa Cruz Biotech, Santa Cruz, CA) and established methods (35). This antibody detects VEGF as a dimer of $43 \mathrm{kDa}$ molecular weight.

\section{Reverse Transcriptase-Quantitative Polymerase Chain Reaction} (RT-qPCR)

Total RNA was extracted from fetal retinal samples using the singlestep acid guanidinium thiocyanate-phenol-chloroform extraction (36) and was then reverse-transcribed using a commercially-available kit (cDNA Archive kit, Applied Biosystems, Foster City, CA). The expression of VEGF isoforms A-D was measured using standard SYBR green I-based RT-qPCR (37).

Isoform-specific primers were designed using the Beacon Design software (Bio-Rad, Hercules, CA): VEGF-A forward: CCTTGCCTTGCTGCTCTAC, reverse: TTCTGCCCTCCTCCTT CTG; VEGF-B forward: GGACAGAGTTGGAAGAGGAGAC, reverse: GGAAGAGCCAGTTGTAAGATGC; VEGF-C forward: CAGACAAGTTCATTCCATTATTAG, reverse:AGTCATCTCCAGC ATCCG; VEGF-D forward: TCCAGACCAACCTTCCATTCAC, reverse: CAGCACACCTTTCTCATTCACC. For quantitative measurements, we developed a standard curve by reverse-transcribing serial dilutions of total RNA from Hep3B cells, an immortalized cell line that constitutively expresses VEGF (38).

The architecture of VEGF-A was analyzed using the databases aceview (19), GeneCard (39), and Ensembl (29), and splice variant-specific amplicons were identified as previously described by Lamani et al. (40). We used forward primers designed specifically for each splice variant: $V_{E G F}$ : GAGCAAGACAAGAAAAATGTG; VEGF $_{165}$ : CAGACTCGCGTTGCAAGATG; VEGF-183: GTATAA GTCCTGGAGCGTTCC; VEGF ${ }_{189}$ : AAGCGCAAGAAATCCCGT CC; and VEGF V06 $_{20}$ TGCTGTCTAATGCCCTGGAG, and combined these with a common reverse primer: GTCTTCACTGGA TGTATTTGAC. PCR products were resolved in agarose gels for qualitative analysis. In some experiments, fetal brain and colonic tissue were included for control.

\section{VEGF $_{121}$ ELISA}

VEGF protein concentrations were initially quantified by ELISA that had a linear range of $15.6-1,000 \mathrm{pg} / \mathrm{ml}$ (R\&D Systems, Minneapolis, $\mathrm{MN}$ ). The commercially available kit was designed to measure VEGF $_{165}$, but per manufacturers information could not distinguish $\mathrm{VEGF}_{165}{ }_{165}$ from $\mathrm{VEGF}_{121}$. Additional analyses were therefore performed on available vitreous samples. VEGF $_{121}$ was measured in vitreous humor using an ELISA kit that includes a capture antibody specific for $\mathrm{VEGF}_{121}$ and does not display cross-reactivity for other splice variants of VEGF (MyBiosource, San Diego, CA). This assay had a linear range of $15.625-1,000 \mathrm{pg} / \mathrm{ml}$. Samples containing VEGF concentrations greater than the upper limit of the assay were retested in dilution. Data were normalized against the total protein concentration in each vitreous sample.

\section{Statistical Methods}

Parametric and nonparametric tests were applied using the the Sigma Stat 3.1.1 software (Systat, Point Richmond, CA). Regression analyses and ANOVA were performed on endogenous control data. For PCR data, crossing-threshold $(\Delta \Delta \mathrm{CT})$ values were compared across experimental groups using analysis of variance or the Kruskal-Wallis $H$-test/ Dunn's multiple post-test. In all tests, $P<0.05$ was accepted as significant.

\section{ACKNOWLEDGMENTS}

The authors thank Curtis Boyd, MD, and staff at Southwestern Women's Options, as well as Mabel Padilla for technical assistance.

\section{STATEMENT OF FINANCIAL SUPPORT}

This study was supported by a grant from the Department of Pediatrics, University of New Mexico (R.K.O.) and the US National Institutes of Health award HD059142 (A.M.).

Disclosure: The authors have no financial or other conflicts to disclose.

\section{REFERENCES}

1. Hartnett ME, Penn JS. Mechanisms and management of retinopathy of prematurity. N Engl J Med 2012;367:2515-26.

2. Hellström A, Ley D, Hansen-Pupp I, et al. New insights into the development of retinopathy of prematurity-importance of early weight gain. Acta Paediatr 2010;99:502-8. 
3. Carlo WA, Bell EF, Walsh MC; SUPPORT Study Group of the Eunice Kennedy Shriver NICHD Neonatal Research Network. Oxygen-saturation targets in extremely preterm infants. N Engl J Med 2013;368:1949-50.

4. Eckert GU, Fortes Filho JB, Maia M, Procianoy RS. A predictive score for retinopathy of prematurity in very low birth weight preterm infants. Eye (Lond) 2012;26:400-6.

5. Carlo WA, Finer NN, Walsh MC, et al. Target ranges of oxygen saturation in extremely preterm infants. N Engl J Med 2010;362:1959-69.

6. Gammons MV, Dick AD, Harper SJ, Bates DO. SRPK1 inhibition modulates VEGF splicing to reduce pathological neovascularization in a rat model of retinopathy of prematurity. Invest Ophthalmol Vis Sci 2013;54: 5797-806.

7. Liu P, Wu D, Zhou W, et al. Association of VEGF gene polymorphisms with advanced retinopathy of prematurity: a meta-analysis. Mol Biol Rep 2012;39:10731-7.

8. Gogat K, Le Gat L, Van Den Berghe L, et al. VEGF and KDR gene expression during human embryonic and fetal eye development. Invest Ophthalmol Vis Sci 2004;45:7-14.

9. Hughes S, Yang H, Chan-Ling T. Vascularization of the human fetal retina: roles of vasculogenesis and angiogenesis. Invest Ophthalmol Vis Sci 2000;41:1217-28.

10. Saint-Geniez M, D’Amore PA. Development and pathology of the hyaloid, choroidal and retinal vasculature. Int J Dev Biol 2004;48:1045-58.

11. Bautch VL. VEGF-directed blood vessel patterning: from cells to organism. Cold Spring Harb Perspect Med 2012;2:a006452.

12. Park JE, Keller GA, Ferrara N. The vascular endothelial growth factor (VEGF) isoforms: differential deposition into the subepithelial extracellular matrix and bioactivity of extracellular matrix-bound VEGF. Mol Biol Cell 1993;4:1317-26.

13. Stone J, Itin A, Alon T, et al. Development of retinal vasculature is mediated by hypoxia-induced vascular endothelial growth factor (VEGF) expression by neuroglia. J Neurosci 1995;15(7 Pt 1):4738-47.

14. Adamis AP, Shima DT. The role of vascular endothelial growth factor in ocular health and disease. Retina 2005;25:111-8.

15. Ferrara N, Keyt B. Vascular endothelial growth factor: basic biology and clinical implications. EXS 1997;79:209-32.

16. Konopatskaya O, Churchill AJ, Harper SJ, Bates DO, Gardiner TA. VEGF165b, an endogenous C-terminal splice variant of VEGF, inhibits retinal neovascularization in mice. Mol Vis 2006;12:626-32.

17. Tischer E, Mitchell R, Hartman T, et al. The human gene for vascular endothelial growth factor. Multiple protein forms are encoded through alternative exon splicing. J Biol Chem 1991;266:11947-54.

18. Curatola AM, Moscatelli D, Norris A, Hendricks-Munoz K. Retinal blood vessels develop in response to local VEGF-A signals in the absence of blood flow. Exp Eye Res 2005;81:147-58.

19. Thierry-Mieg D, Thierry-Mieg J. 2006 AceView: a comprehensive cDNAsupported gene and transcripts annotation. Genome Biol 2006;7:Suppl 1:S12 11-14.

20. Nowak DG, Woolard J, Amin EM, et al. Expression of pro- and antiangiogenic isoforms of VEGF is differentially regulated by splicing and growth factors. J Cell Sci 2008;121(Pt 20):3487-95.

21. Baba T, McLeod DS, Edwards MM, et al. VEGF $165 \mathrm{~b}$ in the developing vasculatures of the fetal human eye. Dev Dyn 2012;241:595-607.

22. Slomiany MG, Rosenzweig SA. Hypoxia-inducible factor-1-dependent and -independent regulation of insulin-like growth factor-1-stimulated vascular endothelial growth factor secretion. J Pharmacol Exp Ther 2006;318:666-75.

23. Kim I, Ryan AM, Rohan R, et al. Constitutive expression of VEGF, VEGFR1, and VEGFR-2 in normal eyes. Invest Ophthalmol Vis Sci 1999;40:211521.

24. Pierce EA, Avery RL, Foley ED, Aiello LP, Smith LE. Vascular endothelial growth factor/vascular permeability factor expression in a mouse model of retinal neovascularization. Proc Natl Acad Sci USA 1995;92:905-9.

25. Pe’er J, Shweiki D, Itin A, Hemo I, Gnessin H, Keshet E. Hypoxia-induced expression of vascular endothelial growth factor by retinal cells is a common factor in neovascularizing ocular diseases. Lab Invest 1995;72: $638-45$.

26. Smith LE. Through the eyes of a child: understanding retinopathy through ROP the Friedenwald lecture. Invest Ophthalmol Vis Sci 2008;49: 5177-82.

27. Alon T, Hemo I, Itin A, Pe'er J, Stone J, Keshet E. Vascular endothelial growth factor acts as a survival factor for newly formed retinal vessels and has implications for retinopathy of prematurity. Nat Med 1995;1: 1024-8.

28. Bossi E, Koerner F. Retinopathy of prematurity. Intensive Care Med 1995;21:241-6.

29. Hubbard TJ, Aken BL, Beal K, et al. Ensembl 2007. Nucleic Acids Res 2007;35(Database issue):D610-7.

30. Mintz-Hittner HA, Kennedy KA, Chuang AZ; BEAT-ROP Cooperative Group. Efficacy of intravitreal bevacizumab for stage 3+ retinopathy of prematurity. N Engl J Med 2011;364:603-15.

31. Patel S, Rowe MJ, Winters SA, Ohls RK. Elevated erythropoietin mRNA and protein concentrations in the developing human eye. Pediatr Res 2008;63:394-7.

32. Watanabe D, Suzuma K, Matsui S, et al. Erythropoietin as a retinal angiogenic factor in proliferative diabetic retinopathy. $\mathrm{N}$ Engl J Med 2005;353:782-92.

33. Giebel SJ, Menicucci G, McGuire PG, Das A. Matrix metalloproteinases in early diabetic retinopathy and their role in alteration of the blood-retinal barrier. Lab Invest 2005;85:597-607.

34. Mititelu M, Chaudhary KM, Lieberman RM. An evidence-based metaanalysis of vascular endothelial growth factor inhibition in pediatric retinal diseases: part 1. Retinopathy of prematurity. J Pediatr Ophthalmol Strabismus 2012;49:332-40.

35. Kurien BT, Scofield RH. Western blotting. Methods 2006;38:283-93.

36. Chomczynski P, Sacchi N. Single-step method of RNA isolation by acid guanidinium thiocyanate-phenol-chloroform extraction. Anal Biochem 1987;162:156-9.

37. Morrison TB, Weis JJ, Wittwer CT. Quantification of low-copy transcripts by continuous SYBR Green I monitoring during amplification. Biotechniques 1998;24:954-8, 960, 962.

38. Semenza GL, Wang GL. A nuclear factor induced by hypoxia via de novo protein synthesis binds to the human erythropoietin gene enhancer at a site required for transcriptional activation. Mol Cell Biol 1992;12:5447-54.

39. Safran M, Chalifa-Caspi V, Shmueli O, et al. Human Gene-Centric Databases at the Weizmann Institute of Science: GeneCards, UDB, CroW 21 and HORDE. Nucleic Acids Res 2003;31:142-6.

40. Lamani E, Wu Y, Dong J, Litaker MS, Acevedo AC, MacDougall M. Tissueand cell-specific alternative splicing of NFIC. Cells Tissues Organs 2009;189:105-10. 